\title{
Neutrinoless double beta decay and QCD running at low energy scales
}

\author{
M. González, ${ }^{1, *}$ M. Hirsch, ${ }^{2, \dagger}$ and S. G. Kovalenko ${ }^{1, \$}$ \\ ${ }^{1}$ Universidad Técnica Federico Santa María, Centro-Científico-Tecnológico de Valparaíso, \\ Casilla 110-V, 2390123 Valparaíso, Chile \\ ${ }^{2}$ AHEP Group, Instituto de Física Corpuscular-C.S.I.C./Universitat de València Edificio de \\ Institutos de Paterna, Apartado 22085, E-46071 València, Spain
}

(Received 27 November 2017; published 5 June 2018)

\begin{abstract}
There is a common belief that the main uncertainties in the theoretical analysis of neutrinoless double beta $(0 \nu \beta \beta)$ decay originate from the nuclear matrix elements. Here, we uncover another previously overlooked source of potentially large uncertainties stemming from nonperturbative QCD effects. Recently perturbative QCD corrections have been calculated for all dimension 6 and 9 effective operators describing $0 \nu \beta \beta$-decay and their importance for a reliable treatment of $0 \nu \beta \beta$-decay has been demonstrated. However, these perturbative results are valid at energy scales above $\sim 1 \mathrm{GeV}$, while the typical $0 \nu \beta \beta$ scale is about $\sim 100 \mathrm{MeV}$. In view of this fact we examine the possibility of extrapolating the perturbative results towards sub-GeV nonperturbative scales on the basis of the QCD coupling constant "freezing" behavior using background perturbation theory. Our analysis suggests that such an infrared extrapolation does modify the perturbative results for both short-range and long-range mechanisms of $0 \nu \beta \beta$-decay in general only moderately. We also discuss that the tensor $\otimes$ tensor effective operator cannot appear alone in the low energy limit of any renormalizable high-scale model and then demonstrate that all five linearly independent combinations of the scalar and tensor operators, which can appear in renormalizable models, are infrared stable.
\end{abstract}

DOI: 10.1103/PhysRevD.97.115005

\section{INTRODUCTION}

From neutrino oscillation experiments it is nowadays well known that at least two neutrinos have nonzero masses. Oscillation experiments, however, cannot decide whether neutrinos are Dirac or Majorana particles. Lepton number violating (LNV) processes have to be studied to explore the neutrino nature [1-3]. Neutrinoless double beta decay $(0 \nu \beta \beta)$, for recent reviews see for instance [4-6], is by far the most powerful available probe of lepton number violation, and its nonobservation allows us to constrain LNV beyond standard model (BSM) physics. From several experimental searches for $0 \nu \beta \beta$ [7-10] the best current bound on the $0 \nu \beta \beta$ half-life, $T_{1 / 2}^{0 \nu}$, comes from the KamLAND-Zen experiment [8],

\footnotetext{
*marcela.gonzalezp@usm.cl

†mahirsch@ific.uv.es

*sergey.kovalenko@usm.cl
}

Published by the American Physical Society under the terms of the Creative Commons Attribution 4.0 International license. Further distribution of this work must maintain attribution to the author(s) and the published article's title, journal citation, and DOI. Funded by SCOAP .

$$
T_{1 / 2}^{0 \nu}\left({ }^{136} \mathrm{Xe}\right) \geq 1.07 \times 10^{26} \text { ys }(90 \% \text { C.L. }) .
$$

From the theoretical perspective, there are two different kinds of contributions to the $0 \nu \beta \beta$ amplitude: the shortrange mechanisms (SRM) [11], which are mediated by heavy particle exchange, and the long-range mechanisms (LRM) [12], in which a light neutrino is exchanged between two pointlike vertices. It has been recently demonstrated that QCD corrections to $0 \nu \beta \beta$ are important, especially in the SRM case [13] due to the presence of the color-mismatch effect and the corresponding mixing of different operators, with numerically very different nuclear matrix elements (NME). This effect leads to differences in the limits on the Wilson coefficients (WC), which amount in some cases up to 3 orders of magnitude. On the other hand, the LRM operate between two different and distant nucleons, so that no color-mismatch appears and only QCD vertex corrections have to be taken into account. Their effect on the extracted limits does not exceed 60\% [14], less than the typical estimate of the uncertainties of the NMEs.

These QCD Renormalization group equation (RGE) results [13,14] are valid for energy scales above $\sim 1 \mathrm{GeV}$, the limit of perturbative QCD. On the other hand, the typical scale of $0 \nu \beta \beta$-decay is about $\sim 100 \mathrm{MeV}$. It is then natural to ask whether these perturbative results still can be considered a reasonable approximation or 
whether they could be drastically affected by some nonperturbative effects. In this paper, we try to address this issue within the semiempirical approach of freezing the running of the strong coupling constant at low energies. Freezing is motivated by some approaches to nonperturbative QCD effects, in particular, by the background perturbation theory (BPTh) [15]. We do not pretend that our work determines the final conclusion about this question, but rather hope that our analysis will allow us at least to visualize this problem, overlooked in the literature, and stimulate efforts for theoretically grounded studies in this direction. The conventional approach relies on the operator product expansion for observables, such as $0 \nu \beta \beta$ half-life, and on a proper matching of the quark- and nucleon-level theories at a certain scale $\mu_{0}$. This is a low energy scale, down to which perturbative QCD for the quark-level theory is valid. In a self-consistent approach both Wilson coefficients and the nucleon matrix elements of the effective operators depend on $\mu_{0}$ in such a way that the effect in any observable should cancel, in theory. Unfortunately, the nucleon matrix elements of $0 \nu \beta \beta$ operators have not been studied yet from this perspective. The hadronization prescriptions existing in the literature (see, for instance, Refs. [16,17]) parametrize the matrix elements in terms of phenomenological nucleon form factors losing connection with QCD and, therefore, a dependence on the matching scale $\mu_{0}$ remains. Presumably, lattice QCD is able to shed light on this issue. Some recent lattice QCD publications about matrix elements of $0 \nu \beta \beta$-operators can be found in Refs. [18-20]. Recently an approach to hadronization in $0 \nu \beta \beta$-decay, which probably suits better the matching with lattice QCD, has been developed in Refs. [21,22] on the basis of chiral effective field theory. However, the issue of the matching-scale dependence still remains unaddressed. This is the situation in which we adopted the idea of freezing. In a sense it can be thought of as a rough modeling of the matching scale dependence of the nucleon matrix elements alleviating the dependence of the $0 \nu \beta \beta$ half-life on this scale.

\section{QCD RUNNING COUPLING CONSTANT IN THE INFRARED LIMIT}

The high energy behavior of QCD is well understood. Thanks to asymptotic freedom, perturbation theory allows an accurate calculation of the running QCD coupling constant $\alpha_{s}(\mu)$. In the infrared region, however, nonperturbative effects become important and lead, in particular, to color confinement at some energy scale below $\sim 1 \mathrm{GeV}$ [23-25]. At such low energies, perturbative QCD (pQCD) suffers a singularity, the so-called Landau pole; i.e., $\alpha_{s}(\mu)$ tends to infinity. This singularity prohibits even a formal extrapolation of the pQCD results to the region below $\sim 1 \mathrm{GeV}$. Unsurprisingly, in the literature there is a plethora of approaches aiming at the extrapolation of the pQCD results towards the infrared (IR) region taking into account at least some nonperturbative effects suggested by QCD. We do not discuss all of these attempts and instead refer to the recent review [25] on this subject.

However, different authors have discussed the possibility; for a list of references see for example [25], that the predictions of QCD can be formulated in terms of a modified $\tilde{\alpha}_{s}(\mu)$, which is finite in the IR regime. Basically, the strong increase of $\alpha_{s}$, predicted by $\mathrm{pQCD}$, is thought to be regularized by nonperturbative effects summed up in an effective $\tilde{\alpha}_{s}(\mu)$, which stops growing at some infrared scale. This IR behavior, dubbed freezing of $\alpha_{s}$ in the literature, is compatible with the lattice QCD simulations [26,27].

To give an example let us consider the BPTh [15] applied to QCD. In this approach the gluon field $A_{\mu}$ is separated into two parts $A_{\mu}=a_{\mu}+B_{\mu}$, the perturbative part $a_{\mu}$, and an effective nonperturbative background field $B_{\mu}$. The BPTh one-loop QCD running coupling constant has been calculated in Refs. [28,29]. It has been shown that the nonperturbative background field generates an effective mass $M_{B}$ for the gluon in such a way that the argument of $\alpha_{s}\left(\mu^{2}\right)$ is replaced by $\mu^{2} \rightarrow \mu^{2}+M_{B}^{2}$. In the UV limit the effects of the background field become negligible, while at low energies it essentially leads to a cutoff in the running. Then, the modified one-loop QCD running coupling constant can be written as

$$
\tilde{\alpha}_{s}\left(\mu^{2}\right)=\frac{\alpha_{s}(\lambda)}{1+\beta_{0} \frac{\alpha_{s}(\lambda)}{4 \pi} \log \frac{\mu^{2}+M_{B}^{2}}{\lambda^{2}}} .
$$

Here, $\beta_{0}$ is the usual $\beta$-coefficient, while the mass parameter, $M_{B}$, is nonuniversal and depends on the specific process in consideration $[29,30]$.

The parameter $M_{B}$ can be related to the mass of the glueball $M_{2 g}\left(0^{++}\right)$[29], a bound state of two gluons connected with the adjoint string $M=M_{B}=M_{2 g}\left(0^{++}\right)=$ $\sqrt{2 \pi \sigma_{a}} \sim 2 \mathrm{GeV}$, or in the case of static $Q \bar{Q}$ potential $M=M_{Q \bar{Q}}=\sqrt{2 \pi \sigma_{f}} \sim 1 \mathrm{GeV}$. In Refs. [31,32] $\alpha_{s}$ has been calculated in the BPTh up to three loops and the value $M_{B}=1.06 \mathrm{GeV}$ has been extracted from the analysis of bottomium fine structure. We mention also [33], quoting $M_{B}=0.95 \mathrm{GeV}$, and [28], which give $M_{B}=2.15 \mathrm{GeV}$.

Below we revisit the QCD corrections to the SRM and LRM of $0 \nu \beta \beta$-decay $[13,14]$ applying Eq. (2) in order to extrapolate the pQCD results towards $\mu \sim 100 \mathrm{MeV}$, the characteristic scale of $0 \nu \beta \beta$-decay. In order to estimate the uncertainty of our results, we do not use some specific value of $M_{B}$ and instead show our results as functions of the calculated value of the "frozen" value of $\alpha_{S}^{F}$, corresponding to $\tilde{\alpha}_{s}\left(\mu^{2}\right)$ for $\mu \leq 0.1 \mathrm{GeV}$. Although not numerically relevant, for completeness we mention that for the normalization of the expression in Eq. (2), we use the experimental value $\alpha_{s}\left(\mu=M_{Z}\right)$ from [34]. 


\section{QCD RUNNING OF WILSON COEFFICIENTS}

Here we apply the IR extrapolation based on freezing, discussed above, to the pQCD results for $0 \nu \beta \beta$-decay derived in Refs. [13,14,35] (and based on [36]).

\section{A. pQCD Wilson coefficients}

First let us recall the $\mathrm{pQCD}$ results for the short-range and long-range mechanisms of $0 \nu \beta \beta$-decay derived in $[13,35]$.

(A) Short-range mechanisms of $0 \nu \beta \beta$-decay are mediated by the exchange of heavy particles of mass $M_{I}$ between two nucleons of the decaying nucleus. At low energy scales $\mu<M_{I}$ this mechanism is described by the low energy effective Lagrangian [11,13],

$$
\mathcal{L}_{\text {eff }}^{0 \nu \beta \beta}=\frac{G_{F}^{2}}{2 m_{p}} \sum_{i, X Y} C_{i}^{X Y}(\mu) \cdot \mathcal{O}_{i}^{(9) X Y}(\mu),
$$

with the complete set of dimension-9 nonequivalent $0 \nu \beta \beta$ operators $[13,35]$,

$$
\begin{gathered}
\mathcal{O}_{1}^{(9) X Y}=4\left(\bar{u} P_{X} d\right)\left(\bar{u} P_{Y} d\right) j, \\
\mathcal{O}_{2}^{(9) X X}=4\left(\bar{u} \sigma^{\mu \nu} P_{X} d\right)\left(\bar{u} \sigma_{\mu \nu} P_{X} d\right) j, \\
\mathcal{O}_{3}^{(9) X Y}=4\left(\bar{u} \gamma^{\mu} P_{X} d\right)\left(\bar{u} \gamma_{\mu} P_{Y} d\right) j, \\
\mathcal{O}_{4}^{(9) X Y}=4\left(\bar{u} \gamma^{\mu} P_{X} d\right)\left(\bar{u} \sigma_{\mu \nu} P_{Y} d\right) j^{\nu}, \\
\mathcal{O}_{5}^{(9) X Y}=4\left(\bar{u} \gamma^{\mu} P_{X} d\right)\left(\bar{u} P_{Y} d\right) j_{\mu},
\end{gathered}
$$

where $X, Y=L, R$ and the leptonic currents are

$$
j=\bar{e}\left(1 \pm \gamma_{5}\right) e^{c}, \quad j_{\mu}=\bar{e} \gamma_{\mu} \gamma_{5} e^{c} .
$$

The QCD corrections induce operator mixing due to the color-mismatch effect, which is equivalent to mixing different Wilson coefficients $C_{i}^{X Y}(\mu)$ in Eq. (3) in the amplitude. This effect has a dramatic impact on the numerical predictions of high-scale models for the $0 \nu \beta \beta$-decay half-life $[13,35]$ due to the significant differences in the numerical values of NMEs of different operators (4)-(8). The QCDcorrected $0 \nu \beta \beta$-decay half-life formula reads [13]

$$
\begin{aligned}
{\left[T_{1 / 2}^{0 \nu \beta \beta}\right]^{-1}=} & G_{1}\left|\sum_{i=1}^{3} \beta_{i}^{(\mathrm{SRM}) X Y}\left(\mu_{0}, \Lambda\right) C_{i}^{X Y}(\Lambda)\right|^{2} \\
& +G_{2}\left|\sum_{i=4}^{5} \beta_{i}^{(\mathrm{SRM}) X Y}\left(\mu_{0}, \Lambda\right) C_{i}^{X Y}(\Lambda)\right|^{2}
\end{aligned}
$$

$G_{1,2}$ are phase-space factors [11,37], and summation over the different chiralities $X, Y=L, R$ is implied. The parameters
$\beta_{i}^{(\mathrm{SRM})}$ are defined in [13] and represent linear combinations of the NMEs of the operators (4)-(8).

(B) Long-range mechanisms describe contributions via light neutrino exchange between two quarks belonging to two different and distant nucleons of a decaying nucleus. One of the vertices connected by the neutrino propagator is the standard $V-A$ vertex while the other is a LNV $\Delta L=2$ BSM vertex originating from some heavy particle exchange. At low energies the BSM vertices are given in terms of the effective operators [35],

$$
\begin{gathered}
\mathcal{O}_{1}^{(6) X}=4\left(\bar{u} P_{X} d\right)\left(\bar{e} P_{R} \nu^{C}\right), \\
\mathcal{O}_{2}^{(6) X}=4\left(\bar{u} \sigma^{\mu \nu} P_{X} d\right)\left(\bar{e} \sigma^{\mu \nu} P_{R} \nu^{C}\right), \\
\mathcal{O}_{3}^{(6) X}=4\left(\bar{u} \gamma_{\mu} P_{X} d\right)\left(\bar{e} \gamma^{\mu} P_{R} \nu^{C}\right)
\end{gathered}
$$

with $X=R, L$. Then the $0 \nu \beta \beta$-decay amplitude is given by the second-order perturbation theory in the effective Lagrangian [12,35],

$$
\mathcal{L}_{\text {eff }}^{d=6}=\frac{G_{F}}{\sqrt{2}}\left(j^{\mu} J_{\mu}^{\dagger}+\sum_{i} C_{i}^{X}(\mu) \mathcal{O}_{i}^{(6) X}(\mu)\right) .
$$

Here the first term is the SM low energy 4-fermion effective interaction of the currents

$$
j^{\mu}=\bar{e} \gamma^{\mu}\left(1-\gamma_{5}\right) \nu, \quad J_{\mu}=\bar{d} \gamma_{\mu}\left(1-\gamma_{5}\right) u .
$$

The QCD-corrected $0 \nu \beta \beta$-decay half-life formula for the LRM reads [14]

$$
\left[T_{1 / 2}^{0 \nu \beta \beta}\right]^{-1}=\sum_{i, X} G_{0 i}\left|\beta_{i}^{(\mathrm{LRM}) X}\left(\mu_{0}, \Lambda\right) C_{i}^{X}(\Lambda)\right|^{2},
$$

where $G_{0 i}$ and (NME) $)_{i}$ are the phase-space factors [38]. The coefficients $\beta_{i}^{(\mathrm{LRM})}\left(\mu_{0}, \Lambda\right)$ involve NMEs of the operators (11)-(13) and the pQCD RGE running parameters. Unlike the analogous $\beta$-coefficients in Eq. (10) they do not mix the NMEs of different operators. This is a drastic difference between the SRM and LRM caused by the absence of the color mismatch in the latter case as demonstrated in Ref. [35].

\section{B. Freezing Wilson coefficients}

The Wilson coefficients $C_{i}$ in Eqs. (3) and (14) are calculable in the framework of a particular underlying model above some high energy scale $\Lambda$. These coefficients can be expressed in terms of the model couplings and heavy particle masses (for a comprehensive analysis see Ref. [35]). At the scale $\Lambda$ the model is matched to the effective theory given by the Lagrangians (3) and (14). Then the Wilson coefficients $C_{i}$ should be RGE evolved, taking into account the QCD loop corrections, down to the 
characteristic scale $\mu_{0}$ of $0 \nu \beta \beta$, typically about $100 \mathrm{MeV}$. References $[13,14]$ stopped the RGE evolution at $1 \mathrm{GeV}$. This kind of truncation is a common practice in the literature applying $\mathrm{pQCD}$ to observable processes. We discuss the crucial question about whether the pQCD results are stable on the way from $1 \mathrm{GeV}$ to $100 \mathrm{MeV}$.

The discussion in Sec. II suggests a method for a rough assessment of the effect of the IR extrapolation by simply freezing the one-loop pQCD running coupling. This amounts to the replacement of $\alpha_{s}(\mu)$ by $\tilde{\alpha}_{s}(\mu)$ from Eq. (2) in the expressions for all the coefficients $\beta\left(\mu_{0}, \Lambda\right)$ in Eqs. (10) and (16). Since $\tilde{\alpha}_{s}(\mu)$ is finite in the IR limit, one can then extend the running down to the required value of $\mu_{0} \simeq 100 \mathrm{MeV}$.

Our main results are then shown in Fig. 1 for the nine SRM coefficients and in Fig. 3 for the six LRM coefficients. Both figures show the change of the WCs with respect to their numerical values calculated without freezing, i.e., $\Delta\left(C_{i}^{A B}\right)=C_{i}^{A B}\left(\alpha_{S}^{F}\right) / C_{i}^{A B}\left(\alpha_{S}(1 \mathrm{GeV})\right)$, as a function of the numerical value of $\alpha_{S}^{F}$. Note that there are only
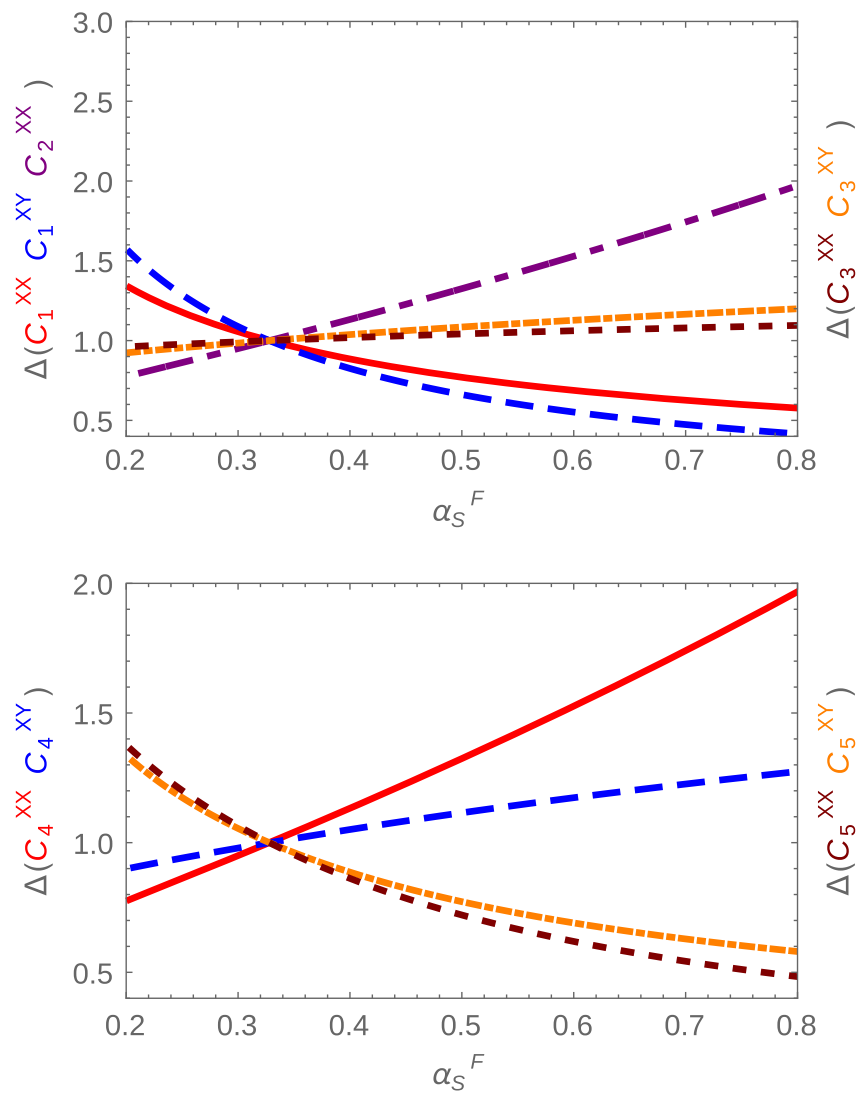

FIG. 1. Relative change of the limit on the short-range coefficients with respect to the frozen value of $\alpha_{S}$ at low energies. Here $\alpha_{S}^{F}$ represents $\tilde{\alpha}_{S}\left(Q^{2}\right)$ for $Q^{2} \leq 0.1 \mathrm{GeV}^{2} . \Delta\left(C_{i}^{A B}\right)$ is calculated normalizing with respect to the value of the coefficient derived without freezing and using an $\alpha_{S}\left(1 \mathrm{GeV}^{2}\right) \simeq 0.32$. Up to $\alpha_{S}^{F} \approx 0.8$, changes of the Wilson coefficients are roughly less than a factor of $\sim 2$. three lines in Fig. 3, because the relative changes of $C_{i}^{L}$ and $C_{i}^{R}$ are the same. Note also that the coefficients are calculated for the particular case of ${ }^{136} \mathrm{Xe}$, but the plots for other isotopes are very similar [since we plot $\Delta\left(C_{i}^{A B}\right)$ ].

As Fig. 1 shows, all nine short-range coefficients change only moderately, when $\alpha_{S}^{F}$ is varied in the window (0.2)(0.8). The two $C_{3}$ are the most stable coefficients, while the other $C_{i}$ change within (less than) a factor of roughly 2 . We point out that it is usually argued in the $0 \nu \beta \beta$-decay community that NME have uncertainties of a factor of roughly 2 as well; thus we call the change of the coefficients under variation of $\alpha_{S}^{F}$ "moderate," since it is of comparable size.

Nevertheless, we mention that for values of $\alpha_{S}^{F} \gtrsim 1$ larger changes of the Wilson coefficients result. Thus, the stability of our extrapolation rests on whether or not the idea of a finite, frozen value of $\alpha_{S}$ is indeed correct.

We also discuss that among the operators in Eq. (5), forming the low energy operator basis, the tensor operators $\mathcal{O}_{2}^{(9)}$ are special in the sense that they can never appear alone, i.e., without $\mathcal{O}_{1}^{(9)}$, in the low energy limit of any renormalizable high-scale model (HSM). This is because renormalizable models do not contain fundamental tensor interactions. Then, at low energies the effective operators $\mathcal{O}_{2}^{(9)}$ can arise only from a Fierz transformation of the scalar operator $\mathcal{O}_{1}^{(9)}$ in the Lorentz and color indices. As was shown in [39] there are five possible linear combinations of these two operators, which can originate from

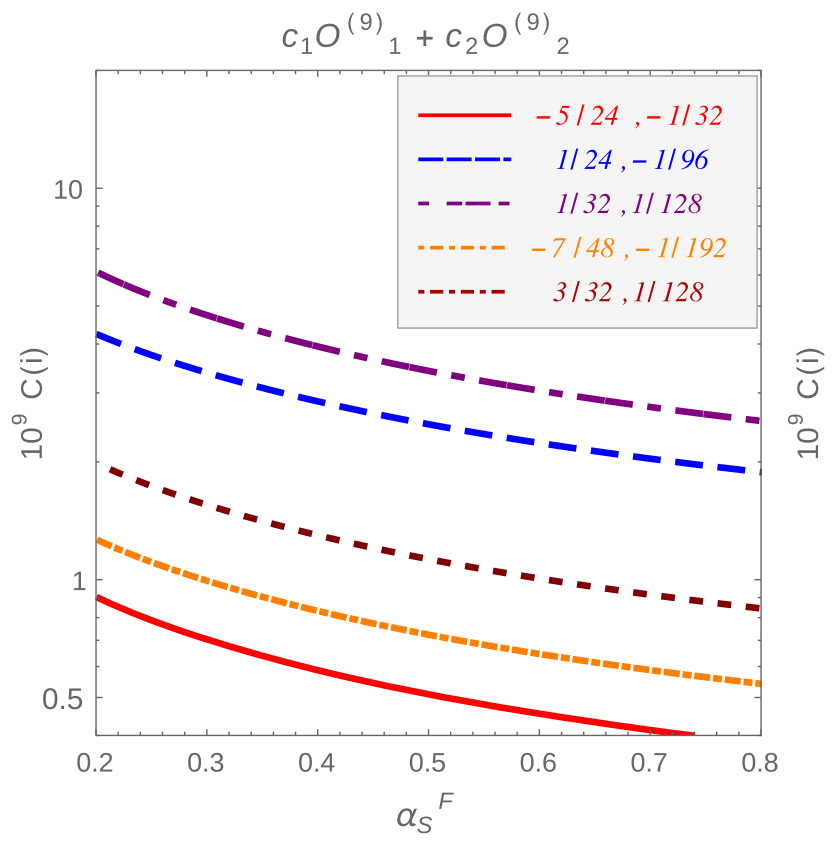

FIG. 2. Limits on the five linearly independent short-range combinations of $c_{1} O_{1}^{(9)}+c_{2} O_{2}^{(9)}$ that appear in the tree-level decomposition of the $0 \nu \beta \beta$-decay operator; see [39]. For a discussion see text. 


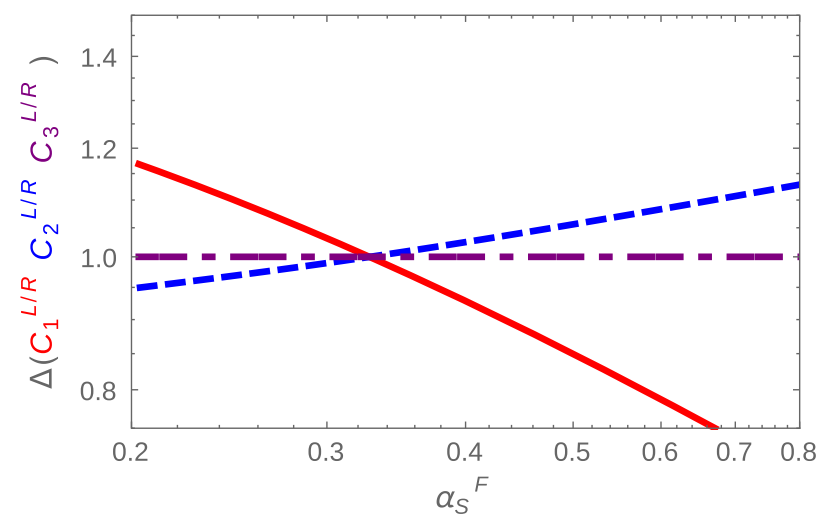

FIG. 3. Relative change of the limit on the long-range coefficients with respect to the frozen value of $\alpha_{S}$ at low energies. Note the change of scale with respect to Fig. 1.

renormalizable high-scale models (for details see Ref. [14]),

$$
\mathcal{O}_{K}^{\mathrm{HSM}}=c_{1} \mathcal{O}_{1}^{(9)}+c_{2} \mathcal{O}_{1}^{(9)}
$$

We show the five combinations of coefficients $c_{1}, c_{2}$ in Fig. 2 together with the corresponding limits on these combinations, again as a function of $\alpha_{S}^{F}$. The plot demonstrates that these limits are stable (within again roughly a factor 2) with respect to variations of $\alpha_{S}^{F}$ for all five cases.

Figure 3, on the other hand, shows that the LRM coefficients are much more stable under variations of $\alpha_{S}^{F}$. Note the change in the scale of the figure relative to Fig. 1. Thus, one can conclude that both, running and freezing, do not play an important role for the long-range part of the $0 \nu \beta \beta$-decay amplitude.

\section{DISCUSSION AND CONCLUSIONS}

We have calculated QCD corrections to $0 \nu \beta \beta$ for both cases, the SRM and LRM, with particular emphasis on the IR behavior of the QCD running coupling. We discussed the regulated form of the QCD running coupling, using background perturbation theory, which introduces the background mass parameter $M_{B}$. In this treatment, the resulting $\tilde{\alpha}_{S}\left(Q^{2}\right)$ freezes at values of $Q^{2}$ smaller than $Q^{2} \leq 0.1 \mathrm{GeV}$. However, since $M_{B}$ in this setup is both a model-dependent as well as a process-dependent parameter, the exact value of $\alpha_{S}^{F}$ is not predicted. We, therefore, showed our results as a function of $\alpha_{S}^{F}$. We conclude that the Wilson coefficients depend only moderately on the exact value of $\alpha_{S}^{F}$ and it seems we can extract reliable limits on these coefficients from $0 \nu \beta \beta$. We noted that in renormalizable ultraviolet completions ("high-scale models") the tensor operator is never generated alone; i.e., it always appears in combination with $O_{1}^{(9)}$. We demonstrated that all combinations of $c_{1} O_{1}^{(9)}+c_{2} O_{2}^{(9)}$ that appear in the (treelevel) decomposition of the $0 \nu \beta \beta$ decay operator are also stable in the above quoted sense.

On the other hand, as a word of caution we need to mention that the idea of a frozen $\alpha_{S}$ at low energy is not at present universally accepted; see the detailed discussion in [25]. Our conclusions remain valid if $\alpha_{S}^{F}$ can be shown to lie definitely below, say, $\alpha_{S}^{F} \lesssim(0.7-0.8)$. For values of $\alpha_{S}^{F}$ much larger than this, our simple-minded treatment cannot be considered to be valid.

Finally, we stress again that the present study does not pretend to present a well theoretically grounded analysis of the nonperturbative QCD effects in the transition region from the quark to the nucleon-level description of $0 \nu \beta \beta$ decay. The problem of the matching, at some low energy scale, of the perturbative QCD results with the effective low energy theory in terms of the nucleon fields remains open and is waiting for its clarification. Future work in this direction would be very valuable.

\section{ACKNOWLEDGMENTS}

This work was supported by the Spanish MICINN Grants No. FPA2014-58183-P, No. FPA2017-85216-P, No. SEV-2014-0398, and No. PROMETEOII/2014/084 (Generalitat Valenciana), and by Fondecyt (Chile) under Grants No. 1150792 and No. 3160642 as well as CONICYT (Chile) Ring ACT 1406 and Basal Grant No. FB0821.
[1] J. Schechter and J. W. F. Valle, Phys. Rev. D 25, 2951 (1982).

[2] M. Duerr, M. Lindner, and A. Merle, J. High Energy Phys. 06 (2011) 091.

[3] M. Hirsch, S. Kovalenko, and I. Schmidt, Phys. Lett. B 642, 106 (2006).

[4] J. Engel and J. Menndez, Rep. Prog. Phys. 80, 046301 (2017).
[5] F. F. Deppisch, M. Hirsch, and H. Päs, J. Phys. G 39, 124007 (2012).

[6] W. Rodejohann, Int. J. Mod. Phys. E 20, 1833 (2011).

[7] J. Albert et al. (EXO-200 Collaboration), Nature (London) 510, 229 (2014).

[8] A. Gando et al. (KamLAND-Zen Collaboration), Phys. Rev. Lett. 117, 082503 (2016); 117, 109903 (2016).

[9] M. Agostini et al., Nature (London) 544, 47 (2017). 
[10] L. Pandola et al. (GERDA Collaboration), in TAUP2017, Sudbury, Canada, https://indico.cern.ch/event/ $606690 /$.

[11] H. Päs, M. Hirsch, H. Klapdor-Kleingrothaus, and S. Kovalenko, Phys. Lett. B 498, 35 (2001).

[12] H. Päs, M. Hirsch, H. V. Klapdor-Kleingrothaus, and S. G. Kovalenko, Phys. Lett. B 453, 194 (1999).

[13] M. González, M. Hirsch, and S. G. Kovalenko, Phys. Rev. D 93, 013017 (2016).

[14] C. Arbeláez, M. González, M. Hirsch, and S. Kovalenko, Phys. Rev. D 94, 096014 (2016).

[15] L. F. Abbott, Nucl. Phys. B185, 189 (1981).

[16] M. Hirsch, H. V. Klapdor-Kleingrothaus, and S. G. Kovalenko, Phys. Rev. D 53, 1329 (1996).

[17] A. Faessler, S. Kovalenko, F. Simkovic, and J. Schwieger, Phys. Rev. Lett. 78, 183 (1997).

[18] A. Nicholson et al., Proc. Sci., LATTICE2016 (2016) 017 [arXiv:1608.04793].

[19] A. Nicholson et al., arXiv:1805.02634.

[20] V. Cirigliano, W. Dekens, M. Graesser, and E. Mereghetti, Phys. Lett. B 769, 460 (2017).

[21] G. Prezeau, M. Ramsey-Musolf, and P. Vogel, Phys. Rev. D 68, 034016 (2003).

[22] V. Cirigliano, W. Dekens, E. Mereghetti, and A. WalkerLoud, arXiv:1710.01729.

[23] G. M. Prosperi, M. Raciti, and C. Simolo, Prog. Part. Nucl. Phys. 58, 387 (2007).

[24] G. Altarelli, Proc. Sci., Corfu2012 (2013) 002 [arXiv: 1303.6065].

[25] A. Deur, S. J. Brodsky, and G. F. de Teramond, Prog. Part. Nucl. Phys. 90, 1 (2016).
[26] A. Cucchieri and T. Mendes, Phys. Rev. D 81, 016005 (2010); I. L. Bogolubsky, E. M. Ilgenfritz, M. Muller-Preussker, and A. Sternbeck, Phys. Lett. B 676, 69 (2009); O. Oliveira and P. J. Silva, Proc. Sci., LAT20092009 (2009) 226 [arXiv:0910.2897]; QCD-TNT092009 (2009) 033 [arXiv:0911.1643]; Phys. Rev. D 86, 114513 (2012).

[27] A. C. Aguilar, D. Binosi, and J. Papavassiliou, J. High Energy Phys. 07 (2010) 002.

[28] Y. A. Simonov, Yad. Fiz. 58, 113 (1995) [Phys. At. Nucl. 58, 107 (1995)].

[29] Y. A. Simonov, Phys. At. Nucl. 74, 1223 (2011).

[30] A. M. Badalian and Y. A. Simonov, Yad. Fiz. 60, 714 (1997) [Phys. At. Nucl. 60, 630 (1997)].

[31] A. M. Badalian, Yad. Fiz. 63, 2269 (2000) [Phys. At. Nucl. 63, 2173 (2000)].

[32] A. M. Badalian and D. S. Kuzmenko, Phys. Rev. D 65, 016004 (2001).

[33] A. M. Badalian, A. I. Veselov, and B. L. G. Bakker, Phys. Rev. D 70, 016007 (2004).

[34] J. Beringer et al. (Particle Data Group), Phys. Rev. D 86, 010001 (2012).

[35] C. Arbeláez, M. González, S. Kovalenko, and M. Hirsch, Phys. Rev. D 96, 015010 (2017).

[36] G. Buchalla, A. J. Buras, and M. E. Lautenbacher, Rev. Mod. Phys. 68, 1125 (1996).

[37] M. Doi, T. Kotani, and E. Takasugi, Prog. Theor. Phys. Suppl. 83, 1 (1985).

[38] M. Doi, T. Kotani, and E. Takasugi, Prog. Theor. Phys. Suppl. 83, 1 (1985).

[39] F. Bonnet, M. Hirsch, T. Ota, and W. Winter, J. High Energy Phys. 03 (2013) 055; 04 (2014) 90. 